\title{
Glukokortikoide: Neubewertung eines Standards
}

\author{
Glucocorticoids: New Evaluation of a Standard
}

\section{Zusammenfassung}

Topische und intern applizierte Glukokortikoide stellen in der täglichen Praxis des Dermatologen die am häufigsten verwendete Substanzklasse dar. Nach ihrer Einführung als Therapeutikum wurden die hochpotenten Kortikoide in den 60er- und 70er-Jahren des vergangenen Jahrhunderts häufig nicht sachgerecht angewendet, so dass unerwünschte Wirkungen zu Verwirrung und Vorurteilen bei Ärzten und Patienten führten, was in einer „Kortiko-Phobie“ bei Teilen der Bevölkerung gipfelte. Trotz der Entwicklung weiterer Immunsuppressiva haben Glukokortikoide auch heute einen großen Stellenwert in der dermatologischen und allergologischen Therapie. Daher benötigen Dermatologen eine intensive Expertise zu Wirkungen, Wirkmechanismen und Indikationen, aber auch der unerwünschten Wirkungen von Glukokortikoiden. Diese Arbeit gibt darüber hinaus einen Überblick über den aktuellen Stand der Empfehlungen zur praktischen Durchführung einer internen und externen Glukokortikoidtherapie.

\section{Abstract}

Topical and systemic glucocorticoids are the most widely used drugs in daily dermatological practice. After their introduction as therapeutical agent in the $1960 \mathrm{~s}$ and $1970 \mathrm{~s}$, inappropriate application led to confusion and prejudice by physicians and patients and finally resulted in "cortico-phobia" in wide parts of the general population. Despite the development of modern immunosuppressive agents, glucocorticoids play an important role in the treatment of allergy and dermatologic diseases. Therefore the dermatologist requires a profound personal experience, and a solid knowledge of mode of action, indications and side effects of glucocorticoids. This review provides a comprehensive overview of the current recommendations on internal and external glucocorticoid treatment.

\section{Einleitung}

Glukokortikoide sind aufgrund ihrer exzellenten Wirksamkeit ein unverzichtbarer Pfeiler im Armentarium der Therapie einer Vielzahl von Dermatosen [1,2]. Jedoch haben Nichtverständnis der Wirkungsweise und häufig nicht individuell an die Dermatose angepasste Wahl des Therapieregimes zu einer „Kortikoidverdrossenheit" geführt, die zusätzlich durch negative Berichterstattung in der Laienpresse verstärkt wurde. Parallel dazu wurde jedoch durch die Entwicklung von Glukokortikoiden der 4. Gene- ration wie z.B. Prednicarbat, das Risiko unerwünschter Wirkungen minimiert.

Die Pharmakotherapie mit Glukokortikoiden erfolgt zum einen als Lokaltherapie, zum anderen als systemische Therapie. Hierbei hat die Lokaltherapie ihren Hauptakzent in der Dermatologie, daneben erfolgen topische Anwendungen am Auge, in den Luftwegen, intraartikulär, intrathekal sowie als Instillation bei entzündlichen Erkrankungen des Kolons. 
Wirkmechanismen und Wirkungen von Glukokortikoiden

Glukokortikoide entfalten ihre Wirkungen modular, d.h. dosisabhängig über genomische und nicht genomische Mechanismen [3].

\section{Genomische Wirkmechanismen}

Am besten untersucht sind derzeit die genomischen Wirkungen. Hierbei binden Glukokortikoide nach Diffusion durch die Zellmembran an ubiquitär exprimierte zytosolische Rezeptoren. Der entstandene aktivierte Steroid-Rezeptor-Komplex bindet nach Translokation in den Zellkern an „glucocorticoid-responsive elements“, welche die Hoch- oder Herabregulierung bzw. Inhibition von Transkriptionsfaktoren, insbesondere „nuclear factor Kappa B“ (NFK B) und ,activating protein 1“ (AP1), Lipokortin I, dem Hemmstoff der Phospholipase A2, sowie diverser proinflammatorischer Zytokine regulieren [4]. Die genomischen Wirkungen haben in allen Dosen therapeutische Relevanz und treten ca. 30 min nach Applikation ein, das Wirkmaximum wird jedoch erst nach 6-8 Stunden erreicht [5].

\section{Spezifische nicht genomische Wirkmechanismen}

In den letzten Jahren wurden für Kortikosteroide Effekte beschrieben, die wahrscheinlich ab Dosen von 0,25-1 mg Prednisolon/KG/die innerhalb von 1-2 min nach Applikation auftreten. Derartig schnelle Effekte sind mit den oben beschriebenen genomischen Wirkmechanismen der Glukokortikoide nicht zu erklären und können auch nicht durch Inhibition der DNA-, RNAoder Proteinbiosynthese gebremst werden. Daher vermutet man membranständige Glukokortikoidrezeptoren, wie sie bereits in Lymphomzellen nachgewiesen wurden, die die Wirksamkeit von Glukokortikoiden im Rahmen von Chemotherapien, wie z.B. dem CHOP-Schema erklären können. Der rasche Wirkeintritt ist am ehesten durch Wirkung auf Second-Messenger-Systeme in Lymphozyten und glatten Muskelzellen zu erklären, wobei für diese Hypothese noch keine gesicherten Daten vorliegen $[5,6]$.

\section{Nicht spezifische, nicht genomische Wirkungen}

In hohen und ultrahohen Dosierungen von $>1 \mathrm{mg} / \mathrm{KG} /$ die bzw. $>1000 \mathrm{mg} /$ die, treten Glukokortikoide in physikochemische Wechselwirkung mit biologischen Membranen, wodurch die Membranfluidität und der Ionenfluss entlang der Membranen vermindert sowie der Phospholipidstoffwechsel beeinflusst wird. In der Folge kommt es zu einem intrazellulären Anstieg der Kalziumkonzentration, die für die Aktivierung und für die Aufrechterhaltung des Aktivierungsstatus von Lymphozyten essenziell ist. Diese In-vitro-Untersuchungen stützen die klinische Erfahrung, dass nur ultrahochdosierte Glukokortikoide in akuten Phasen allergischer Reaktionen sowie schwerer Verlaufsformen von Kollagenosen oder Vaskulitiden rasche Wirkungen zeigen [5].

\section{Wirkungen von Glukokortikoiden}

\section{Antiinflammatorische Wirkung}

Durch die zum Teil durch Inhibition von NFא B und AP-1 mediierte „down regulation“ der proinflammatorischen Mediatoren IL-1, IL-6, IL-12, TNF- $\alpha$ und PAF, werden starke antiinflammatorische
Wirkungen erzielt [7]. Diese werden durch eine vermehrte Expression des „Phospholipase A2 inhibitory protein“, dem Lipocortin I, verstärkt, was zur verminderten Bildung von Arachidonsäure-Produkten führt [8].

\section{Proliferationshemmung}

Durch Hemmung der DNA-Synthese und der dermo-epidermalen Zellteilung ergibt sich der antiproliferative Effekt der Glukokortikoide mit nachfolgender Hemmung der Protein- und insbesondere der Kollagensynthese [9].

\section{Vasokonstriktion}

Die vasokonstriktorischen Mechanismen tragen vermutlich zur Entzündungshemmung bei, z. B. über eine Hemmung der Effekte von Vasodilatatoren wie Histamin, Bradykinin und Prostaglandine [10].

\section{Hemmung der Migration von Immunzellen und Hemmung der Präsentation von MHC-Klasse-II-Molekülen}

Sowohl die Migrationsfähigkeit und Expression von MHC-Klasse-II-Molekülen auf der Oberfläche von Langerhanszellen, als auch die Monozytenchemotaxis werden durch Glukokortikoide gehemmt [11,12].

\section{Wirkstärke von Glukokortikoiden}

Die klassische Einteilung der topisch applizierten Glukokortikoide nach ihrer Wirkstärke wird nach ihrem Einfluss auf Entzündung und Proliferation in Klasse I (schwach), Klasse II (mittel), Klasse III (stark) und Klasse IV (sehr stark) vorgenommen (Tab.1 u.2) [13]. Hierbei wird die Wirkstärke vor allem von der Bindungsstärke des Glukokortikoids an den Glukokortikoidrezeptor bestimmt; jedoch hat auch die Grundlage einen wesentlichen Einfluss, weil Löslichkeit, Konzentration und pH-Wert zu Interaktionen zwischen Vehikel, Wirkstoff und Haut führen können. Demgegenüber korreliert die Wirkstärke intern applizierter Glukokortikoide im „Low-dose“- bis Hochdosisbereich positiv mit der biologischen Halbwertszeit (s. u.). Im Ultra-Hochdosisbereich mit Plasmakonzentrationen $>10^{-4} \mathrm{Mol} / \mathrm{l}$ zeigen sich deutliche, von der klassischen Einteilung abweichende Wirkstärken der Glukokortikoide. Diese Unterschiede sind in Abb.1a u. b zusammengefasst und werden, wie oben beschrieben, durch unterschiedlich starke Interaktionen der Glukokortikoide mit Lipidmembranen und potenziellen membrangebundenen Glukokortikoidrezeptoren erklärt.

\section{Pharmakokinetik und Applikation}

Voraussetzung für die Wirksamkeit glukokortikoidhaltiger Externa ist die Penetration des Wirkstoffs in die Haut, die von folgenden Faktoren beeinflusst wird:

- 1. Hautzustand,

- 2. Grundlageneffekt,

- 3. Wirkstoff,

- 4. Hautalter

Frequenz der Applikation topischer Glukokortikoide

Das Stratum corneum stellt eine Barriere dar, für deren Penetration Glukokortikoide ca. $1 / 4-2$ Stunden benötigen, wobei die erste Aufnahme des Wirkstoffs über die Adnexe geschieht. Gleich- 
Tab. 1 Auswahl topischer Glukokortikoide (in \%) [13]

\begin{tabular}{|c|c|c|c|}
\hline Klasse I (schwach) & Klasse II (mittel) & Klasse III (stark) & Klasse IV (sehr stark) \\
\hline $\begin{array}{l}\text { Hydrokortison } 0,25-1,0 \\
\text { Hydrokortisonacetat } 0,25-1,0 \\
\text { Prednisolon } 0,2-0,4 \\
\text { Clocortolonpivalat } 0,025 \\
\text { Clocortolonpivalat } 0,03 \mathrm{u} \text {. } \\
\text { Clocortolon caproat } 0,03 \\
\text { Triamcinolonacetonid } 0,012 \\
\text { Dexamethason } 0,025\end{array}$ & $\begin{array}{l}\text { Clobetasonbutyrat } 0,05 \\
\text { Hydrokortisonaceponat } 0,1 \\
\text { Hydrokortisonbuteprat } 0,1 \\
\text { Dexamethason } 0,1 \\
\text { Alclometasondipropionat } 0,05 \\
\text { Flumethasonpivalat } 0,02 \\
\text { Triamcinolonacetonid } 0,1 \\
\text { Fluprednidenacetat } 0,1 \\
\text { Clocortolonpivalat } 0,1 \text { und } \\
\text { Clocortolon caproat } 0,1 \\
\text { Hydrocortisonbutyrat } 0,1 \\
\text { Betamethasonbenzoat } 0,025 \\
\text { Fluocortolon } 0,2 \\
\text { Desonid } 0,05-0,1 \\
\text { Betamethasonvalerat } 0,05 \\
\text { Methylprednisolonaceponat } 0,1 \\
\text { Fluocinolonacetonid } 0,01 \\
\text { Desoximethason } 0,05 \\
\text { Prednicarbat } 0,25\end{array}$ & $\begin{array}{l}\text { Mometasonfuroat } 0,1 \\
\text { Betamethasonvalerat } 0,1 \\
\text { Fluticasonpropionat } 0,02-0,05 \\
\text { Halometason } 0,05 \\
\text { Betamethasondipropionat } 0,05-0,64 \\
\text { Fluocortolonpivalat } 0,25 \mathrm{u} \text {. } \\
\text { Fluocortoloncaproat } 0,25 \\
\text { Diflorasondiacetat } 0,05 \\
\text { Desoximethason } 0,25 \\
\text { Fluocinonid } 0,05 \\
\text { Amcinonid } 0,1 \\
\text { Fluocinolonacetonid } 0,01-0,025 \\
\text { Diflucortolonpentanoat } 0,1 \\
\text { Diflucortolonvalerat } 0,1 \\
\text { Halcinonid } 0,1\end{array}$ & $\begin{array}{l}\text { Diflucortolonpentanoat } 0,3 \\
\text { Clobetasolpropionat } 0,05 \%\end{array}$ \\
\hline
\end{tabular}
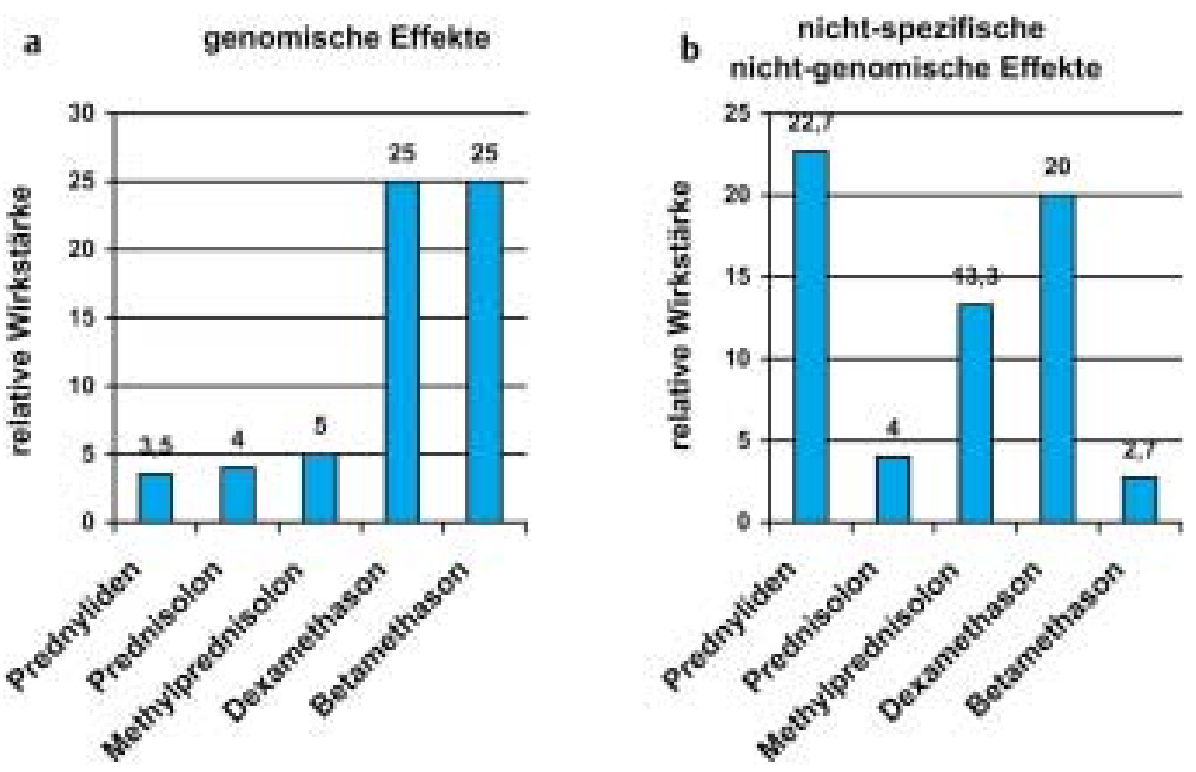

Abb. 1 Relative Wirkstärken verschiedener Glukokortikoide in der Induktion genomischer Effekte und nicht-genomischer Effekte: a Die Daten zu genomischen Effekten wurden von Goodman und Gilbert übernommen und stellen Relativwerte zu Kortisol dar [46]. b Die Daten zu nicht-spezifischen nicht-genomischen Effekten stellen Relativwerte zu Prednisolon dar. Der Wert für Prednisolon wurde mit 4 angesetzt, um einen direkten Vergleich mit klassischen Wirkstärken zu ermöglichen [33].

Tab. 2 Steroid-Wirkprofil

\begin{tabular}{lll}
\hline \multicolumn{1}{c}{ Steroidklasse } & antiinflammatorisch & antiproliferativ \\
\hline 1. schwach & + & - \\
\hline 2. mittel & ++ & + \\
\hline 3. stark & +++ & ++ \\
\hline 4. sehr stark & ++++ & +++ \\
\hline
\end{tabular}

zeitig bilden Glukokortikoide im Stratum corneum ein Reservoir, aus dem sie kontinuierlich freigesetzt werden. Hieraus ergibt sich die Tatsache, dass topische Glukokortikoide meist lediglich einmal täglich appliziert werden müssen, wodurch auch die Compliance verbessert werden kann [14]. Dahingegen wurde bei dreimal täglicher Applikation Tachyphylaxie beobachtet, die durch diskontinuierliche Therapie-Modalitäten verhindert werden kann [15]. Im Gegensatz dazu wurde für exazerbierte Verläufe der Psoriasis ein schnelleres Abheilen bei Applikation von Halcinonid $0,1 \%$ in 8 -stündigen Intervallen beobachtet $[16,17]$. Als weiteres Therapieregime wurde eine wöchentliche „externe Puls-Therapie“ mit Glukokortikoiden der Wirkstärke IV bei pädiatrischen Patienten mit lymphomatoider Papulose als wirksam und gut verträglich beschrieben [18].

\section{Moderne topische Glukokortikoide}

Die Permeation der Haut mit nachfolgender Resorption und systemischer Wirkung sollte möglichst gering gehalten werden. Hier erweisen sich moderne Substanzen mit einem First-Pass-Effekt in der Haut als besonders nebenwirkungsarm. Insbesondere Hautatrophien können durch Verwendung der neu entwickelten 


\section{Stadium madidans}

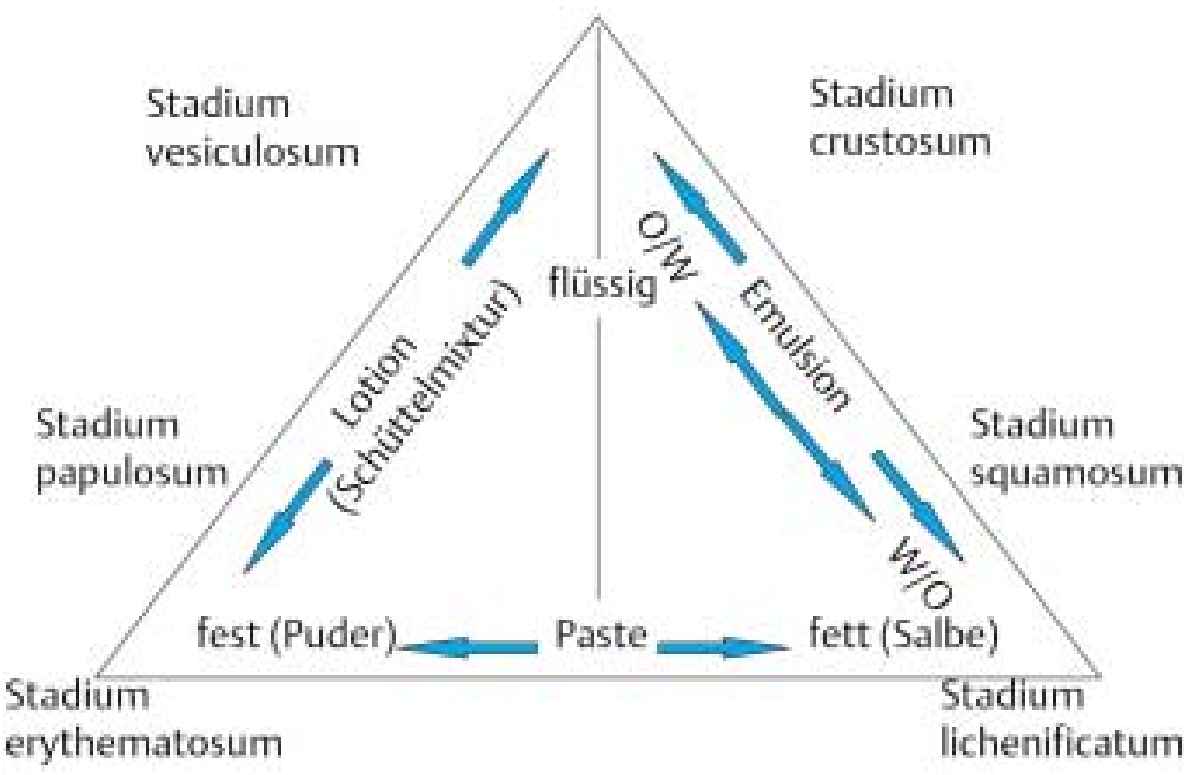

Substanzen Prednicarbat, Mometasonfuorat, Fluticasonpropionat oder Hydrokortisonaceponat vermieden werden [19]. Dahingegen induziert das vor allem in der Asthmatherapie verwendete Budenosid relativ häufig allergische Reaktionen, was auch in geringerem Maße für das hiermit kreuzreagierende Methylprednisolonaceponat zutrifft [20].

\section{Auswahl der Galenik}

Generell muss eine dem Stadium der Dermatose angepasste Galenik gewählt werden, um einen optimalen Therapieerfolg zu gewährleisten. Das Ekzemphasendreieck nach Thoma bietet hierzu einen anschaulichen Überblick (Abb. 2). Die Wirkstoffpenetration hängt zudem stark vom eingesetzten Vehikel ab, d.h., von dessen Fähigkeit, Glukokortikoide freizusetzen.

Okklusion, mit erhöhter Hauthydratation, führt aufgrund der verbesserten Durchlässigkeit des Stratum corneum zu einer Resorptionssteigerung um den Faktor 5-10 und empfiehlt sich daher besonders für die palmoplantare Applikation [21]. Gleichfalls sind Zusätze wie Harnstoff und DMSO Penetrationsbeschleuniger [22]. Des Weiteren haben lipophile Glukokortikoide, wie z. B. in Position 17/21 verestertes Prednisolon, ein besseres Hautpenetrationsvermögen [23].

\section{Anwendungsempfehlungen}

Um eine optimale Glukokortikoidwirkung zu erzielen sollten 1-3 $\mathrm{mg} / \mathrm{cm}^{2}$ appliziert werden, wobei das unterschiedliche Resorptionsverhalten verschiedener Hautareale in Abhängigkeit der Dicke des Stratum corneum beachtet werden muss (Tab. 3) [22]. Daher sollte z. B. der Einsatz von externen Glukokortikoiden der Wirkstärke III-IV im Gesicht, Intertrigines und Genitalbereich vermieden werden [24].
Abb. 2 Ekzemphasendreieck nach Thoma [47]
Tab. 3 Resorptionsverhalten von Hydrokortison auf unterschiedlichen Körperarealen [24]

\begin{tabular}{ll}
\hline Unterarm ventral & 1,0 \\
\hline Unterarm dorsal & 1,1 \\
\hline Fußsohle & 0,14 \\
\hline Fußknöchel lateral & 0,42 \\
\hline Handfläche & 0,83 \\
\hline Rücken & 1,7 \\
\hline Kopfhaut & 3,5 \\
\hline Achselhöhle & 3,6 \\
\hline Stirn & 6,0 \\
\hline Kieferwinkel & 13 \\
\hline Skrotum & 42 \\
\hline
\end{tabular}

Einen weiteren wichtigen Faktor stellt das Hautalter dar. So ist bei Kindern im ersten Lebensjahr die Barrierefunktion nicht voll entwickelt und im Alter muss eine atrophische Epidermis sowie Vergrößerung der Epidermisoberfläche durch Faltenbildung beachtet werden, was zur erhöhten Glukokortikoidaufnahme führt.

Weil schwache externe Glukokortikoide nahezu keine antiproliferative Wirkung entwickeln, sind diese besonders für entzündliche akute Dermatosen geeignet, wohingegen für proliferative Dermatosen oder chronisch lichenifizierte Ekzeme starke bis sehr starke Glukokortikoide gewählt werden sollten (Tab. 4).

Aufgrund der Proliferationskinetik der Epidermiszellen, die morgends ihre höchste und abends die niedrigste Mitoserate aufweisen, empfiehlt sich die Applikation der Glukokortikoide bei hyperproliferativen Dermatosen abends, bei entzündlichen Dermatosen morgens, bzw. tagsüber [25]. 
Tab. 4 Indikationen für eine topische Steroidbehandlung (Auswahl)

\begin{tabular}{|ll}
\hline Diagnose & Wirkstärke \\
\hline Ekzeme im akuten Stadium & I-II \\
\hline chronisch rhagadiforme Ekzeme & III - IV \\
\hline Psoriasis vulgaris (entzündliche Formen) & III - IV \\
\hline Lichen ruber (knotig, verrukös) & III - IV \\
\hline Prurigopapeln (eingeschränkt) & III - IV \\
\hline Mycosis fungoides (UICC Stadium la-IIb) & III - IV \\
\hline Insektenstichreaktionen & I- II \\
\hline Dermatitis solaris & I- II \\
\hline Pityriasis lichenoides & II - III \\
\hline
\end{tabular}

Zur Erzielung eines optimalen Steroid-Effektes, sollten im Sinne einer Tandemtherapie, blande Basiszubereitungen im 12-stündigen Wechsel zur Glukokortikoidapplikation eingesetzt werden. Hiermit wird, neben der pflegenden Wirkung, ein Schutzfilm auf der Haut aufgebaut, der wesentlich dazu beiträgt, zuvor aufgetragene Glukokortikoide im Stratum corneum festzuhalten. Hierzu sind die Grundlagen, die als Träger für das Glukokortikoid eingesetzt werden, besonders geeignet. Für chronische Krankheitsbilder empfiehlt sich neben der Tandemtherapie auch eine Intervalltherapie, bei der sich nach 1-5-tägigen Therapiestößen mit externem Glukokortikoid, ein 1-2-tägiges Intervall der Behandlung mit Basiszubereitungen anschließt.

Des Weiteren empfiehlt sich eine Stufentherapie, bei der nach initialer Therapie mit dem stärksten erforderlichen Glukokortikoid, in Abhängigkeit der Dermatosenrückbildung, ein schrittweiser Übergang auf schwächere Glukokortikoide erfolgen sollte.

Um Reboundphänomene zu verhindern, empfiehlt es sich, die topische Therapie ausschleichend zu beenden. Dies kann durch Reduktion der Wirkstärke, durch Verringerung der täglichen Applikationshäufigkeit bzw. Einschalten mehrtägiger glukokortikoidfreier Intervalle geschehen $[26,27]$. Demgegenüber sollten handelsübliche Glukokortikoidzubereitungen nicht verdünnt werden, da das Wirkprofil aufgrund der möglichen Entwicklung von Substanzinkompatibilitäten, Löslichkeitsproblemen oder Freisetzungsverzögerungen Unsicherheiten aufweist und keine Minimierung unerwünschter Wirkungen eintritt.

Zur Vermeidung von Nebenwirkungen und Tachyphylaxie sollten Glukokortikoide zeitlich begrenzt, und insbesondere Wirkstoffe der Klassen III - IV nur 14-20 Tage bzw. intermittierend eingesetzt werden [19].

\section{Kombinationstherapien}

In einer randomisierten, doppeltverblindeten Multizenterstudie wurde eine gleichstarke antipsoriatische Wirkung von $50 \mu \mathrm{g} / \mathrm{g}$ Calcipotriol und Betamethasonvalerat 0,1\% nachgewiesen, wobei unter der Betamethason-Therapie signifikant seltener Hautirritationen auftraten [28]. In einer weiteren Studie wurde die höhe- re Wirksamkeit einer Kombinationstherapie von $50 \mu \mathrm{g} / \mathrm{g}$ Calcipotriol und Betamethasonvalerat $0,1 \%$ bei deutlich geringeren unerwünschten Wirkungen gegenüber einer Calcipotriol-Monotherapie belegt [29]. Als weitere Kombinationstherapie, mit deutlich kürzerem Behandlungszeitraum bis zum vollständigen Abheilen von Psoriasis-Plaques, wurde eine Hochdosis-Kurzzeittherapie mit Dithranol und Clobetasol-17-Propionat beschrieben [30].

\section{Externe Glukokortikoidtherapie als Alternative zur systemischen Applikation}

In einer Studie an 374 Patienten mit bullösem Pemphigoid wurden die Parameter Überleben, Therapieeffizienz und Rezidivhäufigkeit bewertet. Hierbei fand sich eine niedrigere Sterberate bei gleichzeitig kürzerem Krankenhausaufenthalt und selteneren Komplikationen beim Einsatz von externem Clobetasol im Vergleich zu oral appliziertem Prednisolon. Auch der Zeitraum bis zum Auftreten von Rezidiven war signifikant verlängert, wobei die Unterschiede besonders deutlich bei Patienten mit schwerem Krankheitsverlauf waren [31]. Eine ähnliche Beobachtung wurde bei Patienten mit Polymyalgia rheumatica gemacht, bei denen eine geringere Häufigkeit unerwünschter Wirkungen bei i.m. Applikation von Methylprednisolon im Vergleich zur Standardtherapie mit oralem Prednisolon berichtet wurde. Als Mechanismus wird eine verbesserte Steuerung der Freisetzung der Glukokortikoid-Dosis über 24 Stunden, bei insgesamt steroidsparendem Effekt durch Applikationsformen mit Glukokortikoid-Resorption diskutiert [32].

\section{Systemische Kortilkosteroidtherapie}

Eine Vielzahl dermatologischer Erkrankungen bedarf obligat der systemischen Glukokortikoidtherapie. Hierzu sind insbesondere Autoimmunerkrankungen, allergische Erkrankungen sowie die toxische epidermale Nekrolyse zu zählen. Weiterhin werden systemische Glukokortikoide bei entzündlichen Dermatosen und schweren Ekzemformen mit Befall von mehr als 30\% des Integuments, bei exanthematischem Lichen ruber, Erythrodermien und bei granulomatösen Erkrankungen eingesetzt.

Die Verwendung der Glukokortikoide hat sich nach den allgemeinen Richtlinien der Pharmakodynamik zu orientieren, um unerwünschte Reaktionen zu verhindern, vor allen Dingen im Hinblick auf mögliche Wirkungen auf Natriumausscheidung, Wasserretention und Kaliumverlust. Diese unerwünschte Mineralokortikoidaktivität konnte mit der Entwicklung von Prednison bzw. Prednisolon deutlich verringert werden. Methylprednisolon, Triamcinolon, Dexamethason, Betamethason und Fluocortolon stellen weitere Substanzen zur systemischen Therapie dar, wobei sich Prednisolon als Standardtherapeutikum etabliert hat.

\section{Dosierung interner Glukokortikoide}

In einer Konsensus-Konferenz des Standing Comittee on International Clinical Studies der European League against Rheumatism im April 2001 wurden Größenordnungen von < 7,5 mg/die Pred- 
nisolon als „low dose“, 7,5-30 mg/die als „medium dose“, $30-100 \mathrm{mg} /$ die als „high dose“ sowie $100-250 \mathrm{mg} /$ die als „very high dose" definiert [33]. Puls-Therapien werden mit Dosen $>250 \mathrm{mg} /$ die Prednisolon-Äquivalent an drei aufeinander folgenden Tagen durchgeführt.

Wie oben beschrieben, gelten die klassischen Prednisolon-Äquivalenzen der Wirkstärke von Glukokortikoiden nur für die genomischen Wirkungen und nicht für die nicht-genomischen Wirkungen. Diese unterschiedlichen Äquivalenzen, die lediglich orientierenden Charakter besitzen, wurden in Abb. 1 dargestellt und legen den Einsatz von Dexamethason und Methylprednisolon in der Puls-Therapie nahe $[5,34]$.

\section{Pharmakokinetik und Applikation interner Glukokortikoide}

Glukokortikoide werden zu 90\% aus dem Darm resorbiert, die maximale Plasmakonzentration ist nach ca. 1-2 Stunden erreicht, die optimale Wirkstoffpräsenz wird im Plasma nach 30-60 Minuten erzielt $[35,36]$. Zur intravenösen Applikation werden veresterte Präparate benutzt. Sehr unterschiedlich stellen sich die Plasmaeliminations-Halbwertszeiten (HWZ) dar, die mit der Wirkstärke korrelieren. Die Plasmaeliminations-Halbwertszeit für das kurzwirksame Prednisolon liegt bei 2-3 Stunden, die biologische Halbwertszeit zwischen 18 und 36 Stunden [37]. Die Metabolisierung der Glukokortikoide erfolgt über die Leber, die Ausscheidung der Metaboliten über den Harn. Es sei darauf hingewiesen, dass die Glukokortikoidwirkung aufgrund der genomischen Effekte deutlich länger anhält, als Wirkstoff im Plasma nachzuweisen ist. Eine Reihe verschiedener Einflüsse hat Wirkung auf die HWZ. Eine verkürzte HWZ besteht morgens, bei älteren Menschen sowie Frauen, bei Hyperthyreose, bei gleichzeitiger Einnahme von Barbituraten, Rifampizin, Phenytoin. Eine verlängerte HWZ wird bei Gravidität, Östrogeneinnahme, Leberzirrhose und Myxödem gesehen [38].

Die systemische Glukokortikoidtherapie ist aufgrund der raschen und vollständigen Resorption zumeist oral durchführbar. Hochakute, lebensbedrohliche Krankheitsbilder sowie Störungen im Schluckapparat erfordern eine parenterale Glukokortikoidapplikation.

Die in Tab. 5 genannten Krankheitsbilder und Dosierempfehlungen bezogen auf Prednisolon, stellen orientierende Größenordnungen dar, die jeweils einer individuellen Anpassung im Einzelfall bedürfen [13].

Die Therapiedauer sollte vor allem bei akuten Zuständen nicht länger als 1 Woche dauern, selten 2 Wochen überschreiten. Im Gegensatz dazu ist die Behandlung von z.B. Autoimmunerkrankungen über einen Zeitraum von vielen Wochen bis Monaten anzusetzen. Die Wahl der Erhaltungsdosis hängt gleichfalls vom Krankheitsbild ab und ist unter Umständen über Monate bis Jahre erforderlich, wie z. B. beim systemischen Lupus erythematodes und Pemphigus vulgaris. Durch die Kombination mit Zytostatika kann die Glukokortikoiddosis häufig verringert werden.
Niedrige Erhaltungsdosen sollen dem zirkadianen Rhythmus angepasst werden, also Einnahme der Gesamtmenge vor $8.00 \mathrm{Uhr}$ morgens, damit der adrenale Regelkreis so gering wie möglich belastet wird. Bei Therapie des meist sehr problematischen Asthma bronchiale hat es sich als günstig erwiesen, morgens $2 / 3$ und $1 / 3$ der Tagesdosis am Nachmittag zu verabreichen, wobei häufig die orale Medikation durch Inhalationen zu ersetzen ist. Eine Aufteilung der Prednisolon-Dosis auf 2 Tagesgaben ist aufgrund der Eliminationskinetik auch bei schwer zu beeinflussenden Immunopathien (Pemphigus, Pemphigoid, Dermatomyositis) zu empfehlen. Hierdurch lässt sich die tägliche Gesamtsteroidmenge reduzieren. Eine suffiziente Einmaltagesdosis mit oralen Steroiden ist aufgrund der größeren biologischen Halbwertszeit z.B. mit Fluocortolon oder Methylprednisolon möglich.

\section{Hochdosis-Steroid-Pulstherapie}

Bei lebensbedrohlichen bzw. therapieresistenten Verläufen von Kollagenosen, Vaskulitiden, Pemphigus vulgaris und weiteren entzündlichen Dermatosen können Glukokortikoide in ultrahohen Dosen als Kurzinfusion in 2-6-wöchigen Intervallen zu dauerhaften Remissionen führen. Zusätzlich kann eine Maximierung des Therapieerfolgs durch Kombination mit alkylierenden Substanzen wie Cyclophosphamid, die auch in Kombination mit Glukokortikoiden niedrigdosiert im Intervall appliziert werden können, erreicht werden. Einen Überblick über die bisherigen Erfahrungen gibt Tab. 6 , wobei anzumerken ist, dass es sich hierbei zum überwiegenden Teil um unkontrollierte Studien mit geringen Fallzahlen oder Einzelfallberichte handelt [5].

Als unerwünschte Wirkungen der Pulstherapie wurden neben Exazerbationen bestehender Vorerkrankungen wie arterieller Hypertonie, Diabetes mellitus, gastroduodenaler Ulzera und Infektionen auch Elektrolytentgleisungen, Herzrhythmusstörungen, reversible Geschmacksstörungen, Flush und vegetative Reaktionen beschrieben [5]. Daher sollten unter der Therapie tägliche Kontrollen der Elektrolyte, Glukose und Blutbild sowie des Blutdrucks erfolgen. Als Soorprophylaxe können Antimykotika und bei Hyperglykämie Alt-Insulin verabreicht werden.

\section{I.m. Therapie}

Die intramuskuläre Applikation von wasserlöslichen Kortikosteroiden ist gleichfalls mit sehr gutem Effekt möglich. Im Vergleich zur i.v. Applikation ist der Wirkungseintritt nur wenig verzögert. Dagegen ist die i.m. Anwendung kristalliner Substanzen abzulehnen, weil die Resorptionskinetik großen Schwankungen unterliegt und eine verlässliche Steuerbarkeit nicht zulässt [39]. Zudem ist die Gefahr einer Lipatrophie durch Nachlaufen des Steroids in den Stichkanal nicht auszuschließen.

\section{Dosisreduktion}

Bei langdauernder hochdosierter Kortikosteroidtherapie ist die Dosisreduktion nur in wöchentlichen Intervallen, immer unter Miteinbezug des klinischen Verlaufs, anzuraten, um Exazerbation der Erkrankung, eine Nebennierenrindeninsuffizienz sowie 
Tab. 5 Empfehlungen zur systemischen Glukokortikoidtherapie

\begin{tabular}{|c|c|c|}
\hline Dermatose & Initialdosis & Folgebehandlung tgl. \\
\hline Allergische Urtikaria & $20-60 \mathrm{mg}$ & Reduzierung u. Absetzen nach Verlauf \\
\hline Alopecia areata totalis & $\begin{array}{l}20-40 \text { mg (ca. } 3 \text { Tage) } \\
\text { (u. U. einmalige Pulstherapie } 300 \text { mg) }\end{array}$ & $5-10 \mathrm{mg} \geq 4$ Wochen \\
\hline anaphylaktischer Schock & $\begin{array}{l}\text { Stadium I- II } 250 \text { mg i.v.; Stadium III } 500 \text { mg i.v. } \\
\text { (eventuell mehrmals) }\end{array}$ & \\
\hline $\begin{array}{l}\text { Arzneimittelexantheme } \\
\text { mit Allgemeinsymptomatik }\end{array}$ & $60-80 \mathrm{mg}$ über wenige Tage & Reduzierung u. Absetzen nach Verlauf \\
\hline benignes Schleimhautpemphigoid & 40-120 mg, ca. 3 Wochen & Reduzierung u. Absetzen nach Verlauf \\
\hline bullöses Pemphigoid & 60-100 mg, gelegentlich deutlich höher, 1-3 Wochen & $\begin{array}{l}\text { nach Symptomrückgang vorsichtige Reduzierung } \\
\text { auf individuelle Erhaltungsdosis ca. } 10-30 \mathrm{mg}\end{array}$ \\
\hline Cheilitis granulomatosa & $40-60 \mathrm{mg}$ über $1-2$ Wochen & langsame Dosisreduktion über mehrere Wochen \\
\hline Dermatomyositis & $\begin{array}{l}80-100 \mathrm{mg}, \mathrm{u} \text {. U. deutlich höher, zur Steroideinsparung } \\
\text { Kombination mit Immunsuppressiva }\end{array}$ & $\geq 15$ mg, ggf. über Jahre \\
\hline Ekzeme (Exazerbation, $\geq 30 \%$ Hautbefall) & $40-60-(80) \mathrm{mg}$ wenige Tage & Reduzierung u. Absetzen nach Verlauf \\
\hline eosinophile Fasziitis & $60 \mathrm{mg}$ & langsame Reduktion auf $10-20$ mg \\
\hline Erythema exsudativum multiforme & $60-100 \mathrm{mg} /$ Tag über $1-3$ Wochen & Reduzierung u. Absetzen nach Verlauf \\
\hline Erythema nodosum & $40-80 \mathrm{mg} 1-2$ Wochen & Reduzierung u. Absetzen nach Verlauf \\
\hline Erythrodermie & $80-120 \mathrm{mg} 4-6$ Wochen & ca. $15 \mathrm{mg}$ u. U. Monate \\
\hline Herpes gestationis & $10-40 \mathrm{mg}$ & bis Erscheinungsfreiheit \\
\hline Impetigo herpetiformis & $60-120 \mathrm{mg}$ (Kombination mit AT 10) & Reduzierung u. Absetzen nach Verlauf \\
\hline Lichen ruber exanthematicus & 15 - 40 mg für ca. 3 Wochen & ca. 10 mg über Wochen \\
\hline $\begin{array}{l}\text { Lupus erythematodes } \\
\text { (mit syst. Beteiligung) }\end{array}$ & $\begin{array}{l}\text { entsprechend der Aktivität } 100 \text { - } 200 \text { mg, evtl. aufge- } \\
\text { teilte Tagesgaben (Kombination mit Immunsuppressiva) }\end{array}$ & $\begin{array}{l}\text { über Monate } 5-15 \mathrm{mg} \text {, } \\
\text { Reduzierung nach Aktivität }\end{array}$ \\
\hline Lyell-Syndrom & $100-200-(500) \mathrm{mg}$ für $2-5$ Tage & \\
\hline Morbus Behçet & $60 \mathrm{mg}$ & $\begin{array}{l}\text { Reduzierung nach Verlauf } \\
\text { Erhaltungsdosis } 5-10 \mathrm{mg} \text { über Monate }\end{array}$ \\
\hline Mycosis fungoides Tumorstadium & 40 mg im Rahmen des COPP/MOPP-Schema & jeweils 14 Tage \\
\hline Panarteriitis nodosa & 80 - $120 \mathrm{mg}$, Steroideinsparung mit Azathioprin & langsame Reduktion auf $10-20 \mathrm{mg}$ über Monate \\
\hline Pemphigus vulgaris & 100 - u. U. 400 mg für mehrere Wochen & $\begin{array}{l}\text { langsame Dosisreduktion, ca. } 15-30 \mathrm{mg} \\
\text { (Erhaltung individuell austitrieren) }\end{array}$ \\
\hline Psoriasis pustulosa gen. & $40-80 \mathrm{mg}$ & ca. 4 Wochen \\
\hline Pyoderma gangraenosum & $60-80 \mathrm{mg}$ & ca. $5 \mathrm{mg}$, bis Abheilung \\
\hline Quincke-Ödem & $\begin{array}{l}100-200 \mathrm{mg} \text { Prednisolon einmalig, u. U. wiederholt } \\
\text { bei Schleimhautbeteiligung }\end{array}$ & \\
\hline Relapsing-Polychondritis & $80-100 \mathrm{mg} 2-3$ Wochen & 20 - 30 mg über Wochen \\
\hline Sarkoidose mit Systembefall & $40-60 \mathrm{mg}$ mehrere Monate & ca. 20 mg über Wochen \\
\hline Sézary-Syndrom & Prednisolon 20-40 mg, in Kombination mit Chlorambucil & über Monate \\
\hline Status asthmaticus & $100-250 \mathrm{mg}$ i.v. & $\begin{array}{l}\text { im 6-h-Intervall 50-100 mg i.v. für } 2 \text { Tage, } \\
\text { anschließend Reduzierung nach Verlauf }\end{array}$ \\
\hline Sweet-Syndrom & $60-80 \mathrm{mg}$ ( $1-2$ Wochen) & $15-20 \mathrm{mg}$ (2-6 Wochen) \\
\hline Vasculitis allergica & $20-80 \mathrm{mg}$ & Reduzierung u. Absetzen nach Verlauf \\
\hline
\end{tabular}

Tab. 6 Hochdosis-Steroid-Pulstherapie dermatologischer Erkrankungen (Auswahl) [5]

\begin{tabular}{llll}
\hline Diagnose & Glukokortikoid & Dosierung & Bemerkungen \\
\hline Pemphigus vulgaris & Dexamethason & $100 \mathrm{mg}$, an 3 Tagen & $\begin{array}{l}\text { Komedikation: Cyclophosphamid } \\
500 \mathrm{mg} \text { i.v. an Tag } 1,50 \mathrm{mg} 1 \times \text { tgl. im Intervall }\end{array}$ \\
\hline Pyoderma gangraenosum & Methylprednisolon & $1000 \mathrm{mg}$, an 3-5 Tagen & ggf. Komedikation: Chlorambucil \\
\hline $\begin{array}{l}\text { Dermatomyositis, juvenile } \\
\text { Lichen ruber, exanthematisch }\end{array}$ & Prednisolon & $600 \mathrm{mg}$ an 3 Tagen & \\
\hline
\end{tabular}


ein Kortikosteroid-Entzugssyndrom zu vermeiden. So bedarf es u. U. einer Dosiserhöhung bei schweren Begleitkrankheiten oder Operationen.

\section{Orientierende Absetzmodalitäten}

Ein sofortiges Absetzen kann bei hoher und höchster Steroidtherapie, die für wenige Tage gegeben wurde, erfolgen. Bei 1-2 Wochen dauernder hochdosierter i.v. Therapie, sollte die Verlängerung der Injektionsintervalle auf 24 Stunden und eine letzte Injektion morgens $8.00 \mathrm{Uhr}$ erfolgen. Bei Behandlung über 3-4 Wochen und Dosen über $15 \mathrm{mg}$ täglich um $5 \mathrm{mg}$ bis auf $15 \mathrm{mg}$ reduzieren, ab $15 \mathrm{mg}$ alle $2-3$ Tage um 2,5 mg [40].

Bei einer über Monate, eventuell Jahre durchgeführten Langzeittherapie empfehlen sich folgende Reduzierungsschritte:

- über $30 \mathrm{mg}$ Prednisolon/die Reduzierung um $10 \mathrm{mg}$ alle 2-3 Tage, dann

- bis $15 \mathrm{mg}$ Reduzierung wöchentlich um $5 \mathrm{mg}$,

- ab 15 mg Reduzierung alle 1-2 Wochen um 2,5 mg, dann

- ab $10 \mathrm{mg} /$ die alle 2-8 Wochen um $1 \mathrm{mg}$ reduzieren [40].

\section{Patientenüberwachung bei langfristiger Systemtherapie}

Neben Kontrolluntersuchungen bezüglich der Grunderkrankung sollten 14-tägig Gewicht-, Blutdruck-, Temperaturkontrolle, Erfragung möglicher Befindlichkeitsstörungen, Frage nach zusätzlichen Medikamenten und die Diskussion einer möglichen Steroidreduktion erfolgen. Darüber hinaus sind $1 / 4-$ jährlich hämatologische Untersuchungen, Urinuntersuchung (Kultur, Blutzucker), augenärztliche Kontrolle, sowie alle 6 Monate Knochendichtemessung, Fettstoffwechselanalyse, kardiologische Untersuchung und ggf. Röntgen-Thorax indiziert [40].

\section{Steroidausweis}

Patienten, die einer Langszeitsteroidtherapie bedürfen, sollten einen Kortisonausweis ausgestellt bekommen und diesen stets bei sich führen.

\section{Unerwünschte Wirkungen und Kontraindilkationen}

Eine Übersicht über unerwünschte Arzneimittelwirkungen bei interner und externer Glukokortikoidtherapie geben Tab. 7 u. 8 $[19,41,42]$.

Für die Hormonsubstitutionstherapie bestehen keine Kontraindikationen und für die interne pharmakodynamische Glukokortikoid-Therapie bestehen bei vitalen Indikationen nur relative Kontraindikationen (Tab.9), wohingegen für die externe Glukokortikoidtherapie klare Kontraindikationen bestehen (Tab.10). Daher erfolgt die Therapieentscheidung zum Einsatz von externen Glukokortikoiden nach sorgfältiger Nutzen-Risiko-Analyse.
Tab. 7 Unerwünschte Wirkungen topischer Glukokortikoide an der Haut

\begin{tabular}{l} 
allergisches Kontaktekzem \\
Atrophie \\
Ekzema craquelé \\
Elastose \\
\hline Exazerbation infektiöser Erkrankungen \\
Fettgewebsatrophie \\
Granuloma gluteale infantum \\
Hypertrichose \\
Hypopigmentierung \\
Immunsuppression, lokal \\
Katarakt \\
\hline Komedonen- und Milienbildung \\
okuläre Hypertension \\
rosaceaartige Dermatitis \\
Pseudocicatrices stellaires \\
Purpura \\
Steroidakne \\
Steroidrosazea \\
Steroidulkus \\
Striae \\
\hline Teleangiektasien \\
\hline
\end{tabular}

\section{Impfungen und spezifische Immuntherapie}

Da bei Glukokortikoid-Dosen unter $10 \mathrm{mg}$ keine wesentliche Hemmung der Immunreaktionen auftritt, können im Niedrigdosisbereich Impfungen mit Totimpfstoffen erfolgen, bei Verwendung von Lebendimpfstoffen sollte bei relativer Kontraindikation jedoch eine sorgfältige Risiken-Nutzen-Abwägung erfolgen. Grippeschutzimpfungen werden sogar explizit von der ständigen Impfkommission des Robert-Koch-Instituts empfohlen (Stand Juli 2002).

Analog zu Impfungen, können spezifische Immuntherapien unter niedrig dosierten Glukokortikoiden (bis Prednisolon $10 \mathrm{mg} /$ die) durchgeführt werden.

\section{Glukokortikoide und gastrointestinale Ulzera}

Neuere Untersuchungen zu stress-induzierten Erosionen der gastralen Mukosa zeigen einen protektiven Einfluss endogener Glukokortikoide [43]. Diese Beobachtung steht im Einklang mit bereits 1976 und 1994 veröffentlichten Metaanalysen von bis zu 93 Studien, in denen kein statistisch signifikant erhöhtes Risiko zur Entstehung gastroduodenaler Ulzera (GI-Ulzera) unter interner Glukokortikoidtherapie nachgewiesen werden konnte [44]. Dahingegen findet sich ein deutlich erhöhtes Risiko der Entstehung GI-Ulzera unter der Therapie mit nicht-steroidalen Antiphlogistika (NSAID). Dieses Risiko wird durch eine Kombinationstherapie von NSAID mit Glukokortikoiden noch deutlich erhöht, wobei die Induktion einer Erosion durch das NSAID mit nachfolgender Hemmung der Heilung durch Glukokortikoide vermutet wird [44]. Hieraus lassen sich folgende Empfehlungen für die Praxis ableiten [45]: 
Tab. 8 Unerwünschte Wirkungen bei systemischer Glukokortikoidtherapie

\begin{tabular}{l} 
Alkalose, metabolische \\
Amenorrhö \\
Arthralgien \\
Arthritiden \\
Büffelnacken \\
Kortisonentzugssyndrom \\
Kortisonschäden am Auge \\
Cushing-Syndrom, iatrogenes \\
diabetogene Stoffwechsellage \\
endogener Hypokortizismus \\
exogener Hyperkortizismus \\
hämatologische Veränderungen: Erhöhung der Erythrozytenzahl und \\
Hb-Konzentration, Thrombozytose \\
Hyperphosphatämie \\
Hypokaliämie \\
Impotenz \\
Immunsuppression \\
intestinale Störungen \\
(Kolonperforationen, Pankreatitis) \\
kardiovaskuläre Veränderungen: arterielle Hypertonie, Arteriosklerose, \\
positive Inotropie, verbesserte Ansprechbarkeit kleiner Arterien auf \\
Noradrenalin \\
katabole Stoffwechsellage mit Lipolyse und negativer Stickstoffbilanz \\
(Proteinabbau) \\
Myopathie \\
Natriumretention \\
Osteonekrose \\
Osteoporose \\
Sehnenrupturen \\
Stammfettsucht \\
subaxiale Subluxation der Halswirbelsäule \\
Suppression des adrenalen Regelkreises \\
Wachstumshemmung \\
Wasserretention \\
Wundheilungsstörungen durch Inhibition der Kollagenbiosynthese \\
Zentralnervensystem und Psyche: Senkung der Erregungsschwelle des ZNS, \\
Stimmungsaufhellung bis Euphorie, erhöhte motorische Aktivität, Unruhe, \\
Psychosen \\
\hline intörungen, Stimmungs- und Antriebsschwankungen, Dysphorie, \\
\hline
\end{tabular}

Tab. 9 Relative Kontraindikationen interner Glukokortikoidtherapie

gastrointestinale Ulzera

Infektionskrankheiten

Impfungen mit Lebendimpfstoffen

Myopathie

Psychose, akute

Osteoporose

Schwangerschaft

Stillzeit
Tab. 10 Kontraindikationen einer lokalen Glukokortikoidtherapie

Dermatophytosen
Epizoonosen
Erysipel
Hauttuberkulose
Impetigo contagiosa
Kandidosen
periorale Dermatitis
Rosazea
syphilitische Erscheinungsbilder
virusbedingte Hauterkrankungen

- Eine Ulkusprophylaxe mit Protonenpumpenhemmern oder H2-Blockern ist nur bei positiver Ulkusanamnese oder gleichzeitiger bzw. kürzlich vorausgegangener NSAID-Therapie indiziert.

- Patienten unter Glukokortikoid-Dauertherapie müssen regelmäßig auf die Entstehung eines GI-Ulkus untersucht werden (Blutbild, Stuhl auf Blut, Gastroduodenoskopie).

- Besteht ein GI-Ulkus vor Glukokortikoid-Therapie oder ist es unter der Therapie entstanden, so ist die Glukokortikoid-Therapie nicht kontraindiziert, jedoch müssen engmaschige klinische und gastroskopische Kontrollen erfolgen.

\section{Literatur}

${ }^{1}$ Ring J, Fröhlich HH. Wirkstoffe in der dermatologischen Therapie. Berlin: Springer, 1985

2 Ring J. Angewandte Allergologie. München: Urban und Vogel, 2003

3 Buttgereit F, Wehling M, Burmester GR. A new hypothesis of modular glucocorticoid actions. Athritis Rheum 1998; 41: 761 - 767

${ }^{4}$ De Bosscher K, Van den Berghe W, Haegeman G. Mechanisms of antiinflammatory action and immunosuppression by glucocorticoids: Negative interference of activated glucocorticoid receptor with transcription factors. J Neuroimmunol 2000; 109: 16-22

${ }^{5}$ Reinhold U, Buttgereit F. Hochdosis-Steroid-Pulstherapie gibt es Anwendungen in der Dermatologie? Hautarzt 2000; 51: 738-745

${ }^{6}$ Buttgereit F, Scheffold A. Rapid glucocorticoid effects on immune cells. Steroids 2002; 67: 529-534

${ }^{7}$ De Bosscher K, Van den Berghe W, Vermeulen L et al. Glucocorticoids repress nf-kappa b-driven genes by disturbing the interaction of p65 with the basal transcription machinery, irrespective of coactivator levels in the cell. Proc Natl Acad Sci USA 2000; 97: 3919-3924

${ }^{8}$ Hirata F, Schiffmann E, Venkatasubramanian K et al. A phospholipase A2 inhibitory protein in rabbit neutrophils induced by glucocorticoids. Proc Natl Acad Sci USA 1980; 77: 2533-2536

${ }^{9}$ Hein R, Krieg T. Wirkungen von Kortikoiden auf menschliche Fibroblasten in vitro. Z Haut- u. Geschlk 1989; 64: 13 - 17

${ }^{10}$ Altura B. Role of glucocorticoids in local regulation of blood flow. Am J Physiol 1966; 211: $1393-1397$

${ }^{11}$ Walther A, Riehemann K, Gerke V. A novel ligand of the formyl peptide receptor: Annexin I regulates neutrophil extravasation by interacting with the fpr. Mol Cell 2000; 5: 831 - 840

${ }^{12}$ Cumberbatch M, Dearman R, Kimber I. Inhibition by dexamethasone of langerhans cell migration: Influence of epidermal cytokine signals. Immunopharmacology 1999; 41: 235-243

${ }^{13}$ Niedner R. Hautkrankheiten. In: Kaiser H, Klay HK (Hrsg). Cortisontherapie. Stuttgart: Thieme, 1997

${ }^{14}$ Lagos BR, Maibach HI. Frequency of application of topical corticosteroids: An overview. Br J Dermatol 1998; 139: 763 - 766

${ }^{15}$ Du Vivier A. Tachyphylaxis to topically applied steroids. Arch Dermatol 1976; $112: 1245-1248$ 
${ }^{16}$ Frederiksson T, Lassus A, Bleeker J. Treatment of psoriasis and atopic dermatitis with halcinonide cream applied once and three times daily. Br J Dermatol 1980; 102: 575-577

${ }^{17}$ Sudilovsky A, Muir JG, Bocobo FC. A comparison of single and multiple applications of halcinonide cream. Br J Dermatol 1981; 20: 609-613

18 Paul MA, Krowchuk DP, Hitchcock MG et al. Lymphomatoid papulosis: Successful weekly pulse superpotent topical corticosteroid therapy in three pediatric patients. Pediatr Dermatol 1996; 13: 501-506

${ }^{19}$ Brazzini B. Pimpinelli N. New and established topical corticosteroids in dermatology clinical pharmacology and therapeutic use. Am J Clin Dermatol 2002; 3: 47-58

${ }^{20}$ Corazza M, Virgili A. Allergic contact dermatitis from a-methylprednisolone aceponate and budenoside. Contact Dermatitis 1998; 38: $356-357$

${ }^{21}$ Feldmann R, Maibach H. Penetration of 14C-hydrocortisone through normal skin: The effect of stripping and occlusion. Arch Dermatol 1965; 91: 664-666

22 Wester R, Buchs D, Maibach H. In vivo percutaneous absorption of hydrocortisone in psoriatic patients and normal volunteers. J Am Acad Dermatol 1983; 8: 645-647

${ }^{23}$ Hevert F, Schiffmann I, Busch B, Rozman T. Kortikoid Ester. Dtsch Derm 1989; 37: $678-683$

24 Feldmann R, Maibach HI. Regional variation in percutaneous penetration of $14 C$ cortisol in man. J Invest Dermatol 1967; 48: 181- 183

${ }^{25} \mathrm{Schell} \mathrm{H}$, Hornstein O, Engelmann W et al. Evidence of diurnal variation of human epidermal cell proliferation. 11. Duration of epidermal DNA synthesis. Arch Derm Res 1981; 271: 49-53

${ }^{26}$ Pflugshaupt C. Diskontinuierliche topische Kortikoidtherapie. Z Hautu. Geschlkl 1983; 148: 1229-1235

${ }^{27}$ Du Vivier A, Phillips H, Hehir M. Applications of glucocorticosteroids. The effects of twice daily vs once-every-other-day applications on mouse epidermal cell DNA synthesis. Arch Dermatol 1982; 118: 305-308

28 Molin L, Cutler T, Helander I et al. Comparative efficacy of calcipotriol (mc903) cream and betamethasone 17-valerate cream in the treatment of chronic plaque psoriasis. A randomized, double-blind, parallel group multicentre study. Calcipotriol study group. Br J Dermatol 1997; 136: 89-93

${ }^{29}$ Kragballe K, Barnes L, Hamberg K et al. Calcipotriol cream with or without concurrent topical corticosteroid in psoriasis: Tolerability and efficacy. Br J Dermatol 1998; 139: 649-654

${ }^{30}$ Swinkels O, Prins M, Kucharekova M et al. Combining lesional shortcontact dithranol therapy of psoriasis with a potent topical corticosteroid. Br J Dermatol 2001; 146: 621 -626

${ }^{31}$ Joly P, Roujeau J, Benichou J et al. A comparison of oral and topical corticosteroids in patients with bullous pemphigoid. N Engl J Med 2002; 346: $321-327$
${ }^{32}$ Desgupta BDA, Panayi GS, Fernandes L. An initially double-blind controlled 96 week trial of depot methylprednisolone against oral prednisolone in the treatment of polymyalgia rheumatica. $\mathrm{Br} \mathrm{J}$ Rheumatol 1998; 37: 189-195

${ }^{33}$ Buttgereit F, da Silva JA, Boers M et al. Standardised nomenclature for glucocorticoid dosages and glucocorticoid treatment regimens: Current questions and tentative answers in rheumatology. Ann Rheum Dis 2002; 61: 718-722

${ }^{34}$ Buttgereit F, Brand MD, Burmester GR. Equivalent doses and relative drug potencies for non-genomic glucocorticoid effects: A novel glucocorticoid hierarchy. Biochem Pharmacol 1999; 58: 363-368

35 Bergrem H, Grottum P, Rugstadt H. Pharmacokinetics and protein binding of prednisolone after oral and intravenous administration. Eur J Clin Pharmacol 1983; 24: 415-419

${ }^{36}$ Gupta S, Teng R, Tuntland T et al. Pharmacokinetics and bioavailability of prednisolone and prednisone in healthy volunteers following oral and intravenous administration. Pharm Res 1988; 5: 158-164

${ }^{37}$ Frey B, Frey F. Clinical pharmacokinetics of prednisone and prednisolone. Clin Pharmacokinet 1990; 19: 126-146

${ }^{38}$ Kaiser H, Kley H. Pharmakokinetik der Corticoide. In: Kaiser H, Kley H (Hrsg). Cortisontherapie. Stuttgart: Thieme, 1997

${ }^{39}$ Hatz HJ. Glucocorticoide. Stuttgart: Wiss. Verlagsgesellschaft, 1998

${ }^{40}$ Kaiser H, Kley H. Allgemeine Regeln für die Corticoid-Pharmakotherapie. In: Kaiser H, Kley H (Hrsg). Cortisontherapie Stuttgart: Thieme, 1997

${ }^{41}$ Neumann F, Schenck B, Schleusener H, Schweickert HU. Pharmakotherapie mit Hormonen. In: Forth W, Henschler D, Rummel W, Starke K (Hrsg). Allgemeine und spezielle Pharmakologie und Toxikologie. Mannheim, Leipzig, Wien, Zürich: Spektrum Verlag, 1992

42 Iven $\mathrm{H}$, Korting $\mathrm{H}$. Innerliche Behandlung von Dermatosen. In: BraunFalco O, Plewig G, Wolff H (Hrsg). Dermatologie und Venerologie. Berlin, Heidelberg: Springer, 1994

${ }^{43}$ Filaretova LP, Filaretov AA, Makara GB. Corticosterone increase inhibits stress-induced gastric erosions in rats. Am J Physiol 1998; 274: $1024-1030$

${ }^{44}$ Conn H, Poynard T. Corticosteroids and peptic ulcer: Meta-analysis of adverse events during steroid therapy. J Intern Med 1994; 236: 619-632

45 Del Valle J. Peptic ulcer and related diseases. In: Braunwald E, Fauci AS, Kasper DL, Hauser SL, Longo DL, Jameson JL (Eds). Harrisons internal medicine. New York: McGraw-Hill, 2001

${ }^{46}$ Haynes R, Lorner J. Adenocortical steorids and their synthetic analogues. In: Hardman J, Limbird L (Eds). Goodman and Gilbert's pharmacological basis of therapeutics. New York: McGraw-Hill Companies, 2001

47 Thoma K. Grundlagen und ihre Funktionen in der externen Therapie der Haut. In: Thoma K. Dermatika. München: Werke- und Vertriebsgesellschaft Deutscher Apotheker, 1983 